\title{
BMJ Open Discontinuation of infliximab therapy in patients with Crohn's disease in sustained complete remission (the STOP IT study): protocol for a double- blind, randomised, placebo-controlled, multicentre trial
}

\author{
Sine Schnoor Buhl, ${ }^{1}$ Casper Steenholdt, ${ }^{1}$ Jørn Brynskov, ${ }^{1}$ \\ Ole Østergaard Thomsen, ${ }^{1}$ Klaus Bendtzen, ${ }^{2}$ Mark Andrew Ainsworth ${ }^{1}$
}

To cite: Buhl SS,

Steenholdt C, Brynskov J et al. Discontinuation of infliximab therapy in patients with Crohn's disease in sustained complete remission (the STOP IT study): protocol for a double-blind, randomised, placebocontrolled, multicentre trial. BMJ Open 2014;4: $\mathrm{e} 005887$. doi:10.1136/bmjopen-2014005887

- Prepublication history for this paper is available online. To view these files please visit the journal online (http://dx.doi.org/10.1136/ bmjopen-2014-005887).

Received 8 June 2014 Revised 1 November 2014 Accepted 12 November 2014

\section{CrossMark}

\section{${ }^{1}$ Department of \\ Gastroenterology, Herlev Hospital, Herlev, Denmark ${ }^{2}$ Institute for Inflammation Research, Copenhagen University Hospital, Rigshospitalet, Copenhagen, Denmark}

Correspondence to Dr Sine Schnoor Buhl; sine_buhl@hotmail.com

\section{ABSTRACT}

Introduction: Infliximab (IFX), a monoclonal chimeric antibody against tumour necrosis factor (TNF) $\alpha$, is effective for induction and maintenance of remission in moderate to severe Crohn's disease. Discontinuation of IFX maintenance therapy in patients in remission should be considered in order to reduce the potential long-term side effects and lower costs.

Methods and analysis: This is a prospective, double-blind, randomised, placebo-controlled, multicentre study of patients with luminal Crohn's disease who have been treated with IFX for at least 1 year and are in sustained complete clinical, biochemical and endoscopic remission (ie, Crohn's Disease Activity Index (CDAl) score $<150$, complete mucosal healing and biochemical markers of inflammation within the normal range). These patients are randomised to receive placebo infusions or continue IFX maintenance therapy. The primary end point is the proportion of patients in maintained remission after 48 weeks (def. CDAl <150).

Ethics and dissemination: It is estimated that the knowledge gained about how to optimally handle patients with Crohn's disease in complete long-term sustained remission on IFX is proportionate to the risks and inconveniences related to participation in this study. Prolonged exposure to IFX may cause severe side effects and increased risk of malignancies. Conversely, IFX discontinuation should not unnecessarily create a high risk of relapse. Thus, empirical evidence is needed concerning the safety of discontinuing IFX once a patient exhibits sustained remission. Study results will be published in an English language scientific medical journal. The study is approved by the Danish Medicines Agency (EudraCT-number: 2012-002702-51) and the Regional Ethics Committee of Region Hovedstaden Denmark (Approval-number: H-4-2012-099). The project is reported to the Danish Data Protection Agency (ID-number: 2007-58-0015/HEH.750.89-27), registered at Clinicaltrials.gov, and monitored by independent

\section{Strengths and limitations of this study}

- A state-of-the-art double-blind, randomised, placebo-controlled clinical trial. Previous studies are of retrospective origin, or post hoc analyses of prospective trials. Only two prospective observational studies have been done and these were performed without control groups.

- All evaluations are done by clinically validated scoring systems according to clinical trial practice (eg, Crohn's Disease Activity Index, CDAl).

- Data analyses are not blinded.

- The selected patient population is narrow; consequently, the results can only be extrapolated to a fraction of patients on IFX maintenance therapy.

GCP units for the University of Copenhagen, Odense and Aarhus. The current approved protocol is V.3.2, dated 1 June 2014.

Trial registration number: http://clinicaltrials.gov/ show/NCT01817426.

\section{INTRODUCTION}

Biological agents targeting tumour necrosis factor (TNF) $\alpha$ are effective in inducing and maintaining remission in patients with moderate-to-severe luminal and fistulising Crohn's disease. ${ }^{1-4}$ The chronic nature of Crohn's disease necessitates long-term TNF-inhibiting (TNFi) maintenance treatment. However, the potentially severe side effects such as infections, infusion reactions and increased risk of neoplasia, ${ }^{3}$ along with the high economic cost of the treatment, warrant the exploration of strategies for 
discontinuing TNFi among subgroups of patients in long-term sustained remission.

It is generally accepted that TNFi should not be discontinued in patients without complete remission. However, recent international guidelines conclude that existing data are insufficient to make firm recommendations on when and in whom to stop TNFi treatment. ${ }^{3-7}$ In contrast, several European countries, for example, UK and DK, now recommend re-evaluation of disease activity after 1 year of TNFi therapy to determine if the treatment should be discontinued.

The discontinuation decision is typically made from an individual judgement of benefits versus risks. ${ }^{5} 6$ Concerns related to the discontinuation of TNFi include the risk of disease flare, the risk of infusion reactions at re-initiation, and the possibility of reduced future medical treatment options. ${ }^{5}{ }^{8-11}$ However, early treatment becomes more common and the long-run safety of TNFi has been questioned and its use is therefore not likely to be continued throughout the lives of all patients with Crohn's disease who have obtained clinical remission. Consequently, there is a need for new and evidence-based therapeutic strategies for safe discontinuation of TNFi.

Recently, prospective observational data from the STORI study of 115 patients with luminal Crohn's disease found that $56 \%$ of patients maintained remission 1 year after discontinuation of infliximab (IFX) ${ }^{12}$ In a Hungarian prospective observational study of 121 patients with clinical remission, $45 \%$ had relapse within 1 year after cessation. ${ }^{13}$ Several retrospective studies reported similar relapse rates of 55-85\%. ${ }^{6}$ Consistent with these data, we have reported that $61 \%$ of patients with Crohn's disease, who discontinued IFX while in steroid-free IFX-induced remission, maintained remission for 1 year; indeed, half of the patients remained in remission after nearly 2 years (median 680 (412-948) days). ${ }^{14}$ Taken together, current data indicate that the disease course in patients who discontinue IFX while in clinical remission follow the natural history of Crohn's disease; ${ }^{15}$ prior effective treatment with IFX therefore does not appear to impose a subsequent diseasemodifying effect. It has been suspected that the response to retreatment with IFX in case of relapse after IFX withdrawal may be lost. ${ }^{16}$ However, data from the STORI study and from our centre suggest that patients may respond well to retreatment with IFX at relapse. ${ }^{12} 14$

The term 'remission' is not well defined and may incorporate one or more features such as clinical remission, as assessed by Crohn's Disease Activity Index (CDAI) score, biochemical remission, endoscopic remission, etc. Patients who respond to TNFi therapy, clinical, biochemical and endoscopic, are considered to be in complete remission (typically defined as CDAI score $<150^{17}$ and no other signs of disease activity). Thus, the STORI study identified predictors of relapse, including certain features as well as objective biochemical and endoscopical markers of disease activity. The following risk factors were associated with relapse: (1) male sex; (2) absence of surgical resection; (3) corticosteroid use between 6 and 12 months before discontinuation of IFX; (4) IFX trough level $\geq 2 \mu \mathrm{g} / \mathrm{mL}$ at time of discontinuation of IFX; (5) Crohn's Disease Endoscopic Index Score (CDEIS) $>0$; (6) leucocyte counts $>6.0 \times 10^{9} / \mathrm{L} ;(7) \mathrm{C}$ reactive protein $(\mathrm{CRP})$ $\geq 5.0 \mathrm{mg} / \mathrm{L}$; (8) haemoglobin $\leq 145 \mathrm{~g} / \mathrm{L}$ and (9) faecal calprotectin $\geq 300 \mu \mathrm{g} / \mathrm{g} .{ }^{12}$ A complete model and a simplified model describing the risk of relapse with respect to the number of risk factors present were proposed. Interestingly, patients with $\leq 3$ risk factors in the complete model, and $\leq 2$ risk factors in the simplified model, had a very low risk of relapse of approximately $10 \%$ at 1 year after IFX discontinuation, as compared to a relapse rate of approximately $60 \%$ in those patients with a higher number of risk factors. Thus, degrees of remission might predict the outcome after discontinuation of IFX. Similar to these low-risk patients, relapse rates among patients with ongoing IFX therapy are $13 \%$ per year. ${ }^{18}$

Serum concentrations of TNFi in individual patients vary despite the same dosing. ${ }^{19}{ }^{20}$ At initiation of treatment, high concentrations of TNFi immediately prior to the next administrations, that is, trough levels of the drug, are associated with maintenance of clinical remission. ${ }^{16}{ }^{19-21}$ In addition, repeated infusions/injections of TNFi might result in the patient forming an antibody against the drug (anti-TNFi Ab). Development of anti-IFX $\mathrm{Ab}$ is generally associated with loss of response. ${ }^{16} 192022$ However, patients in complete remission, with high levels of anti-TNFi Ab and low levels of TNFi, are functionally in low drug exposure and might be able to tolerate cessation. However, patients in complete remission during TNFi therapy, with functionally low drug exposure (low levels of TNFi with or without high levels of anti-TNFi Ab), might be able to tolerate cessation. Thus, monitoring patients in remission for circulating levels of functional TNFi as well as for anti-TNFi Ab is warranted to allow optimal individual treatment. Owing to methodological limitations, for example, cross-binding of drug and antidrug $\mathrm{Ab}$ in commonly used binding assays such as ELISA, levels are conventionally measured as trough levels. However, it is highly likely that this is not the optimal time point for assessments of rational therapeutic management.

As described above, there is a need to investigate how to manage patients with Crohn's disease in long-term sustained complete remission. To date, there is little evidence whether IFX can be discontinued favourably and safely in this selected patient group. In prior studies, patients in remission who discontinued IFX therapy faced a higher risk of relapse than patients who continued therapy. However, data suggest that patients with very few risk factors (ie, in complete remission) might have almost similar relapse rates as if IFX therapy were maintained. The intention of this study is to examine whether IFX can be discontinued safely in the complete-remission patient group. To form a rational and efficient management algorithm, we will seek predictors of outcomes after discontinuation. Further, we 
hypothesise that incorporation of measurements of blood levels of IFX and anti-IFX Ab is clinically relevant. A prospective controlled trial is suitable to investigate the optimal management in individual patients and to establish recommendation regarding withdrawal of IFX.

\section{METHODS AND ANALYSIS}

Study design and patients

This study is a prospective, double-blind (patient and physician), randomised, placebo-controlled, multicentre study. A study algorithm is shown in figure 1.

Adult patients (age $\geq 18$ years) with luminal Crohn's disease defined by standardised diagnostic criteria ${ }^{23}$ in sustained complete remission on IFX maintenance therapy (def. infusions every $6-10$ weeks, $5-10 \mathrm{mg} / \mathrm{kg}$ ) with a treatment length of a minimum 12 months are eligible. Complete remission is defined as a CDAI score $<15017$, no signs of inflammation on biochemical parameters (normal CRP, leucocytes, haemoglobin $(\mathrm{Hb})$ and albumin), and no other marks of disease activity, either from endoscopic examination (Simple Endoscopic Score for Crohn's Disease (SES-CD) score 0-2) ${ }^{24} 25$ and/or by MRI (defined by no signs of disease activity when evaluated by a trained radiologist). ${ }^{26}{ }^{27}$ Sustained remission is defined as a clinical judgement of the disease to be stable at two consecutive treatment visits (corresponding two scheduled IFX infusions) and no use of oral steroids within 3 months prior to inclusion. Concomitant therapy with immune suppressants, except steroids, is allowed. The dosage and frequency must have been stable 3 months prior to inclusion, and must remain stable throughout the study period.
Exclusion criteria include the initial indication for IFX being predominantly fistulising perianal disease, ongoing fistulising disease, and pregnancy or lactation. Further, in case of any contraindications for continuing IFX treatment, including prior acute or delayed infusion reaction to a TNF-inhibiting agent, former malignancy, moderate to severe heart disease, any active infection requiring parenteral or oral antibiotic treatment, known infection with tuberculosis, HIV or hepatitis virus, the patient cannot be included.

\section{Course of study}

The course of this study is given in table 1 together with the study algorithm in figure 1 . The total study length is 48 weeks.

The screening visit is defined as clinical remission and 12 months of scheduled IFX therapy minus the interval of regular visits. At the screening visit, biochemical parameters are assessed. Evaluation of mucosal healing is planned before the next visit; endoscopy for patients with colic/ileocolic Crohn's disease; MRI for patients with small bowel Crohn's disease and for patients with fistulising Crohn's disease. The assessed biochemical parameters are: haemoglobin, leucocyte count, platelets, CRP, creatinine, alanine aminotransferase (ALAT), bilirubin, albumin and faecal calprotectin.

The inclusion visit is defined as the time of the next scheduled IFX infusion after the screening visit, thus resulting in an inclusion date after a minimum 12 months of IFX maintenance therapy.

All patients will be graded for disease activity in accordance with CDAI, ${ }^{28}$ Work Productivity and Activity

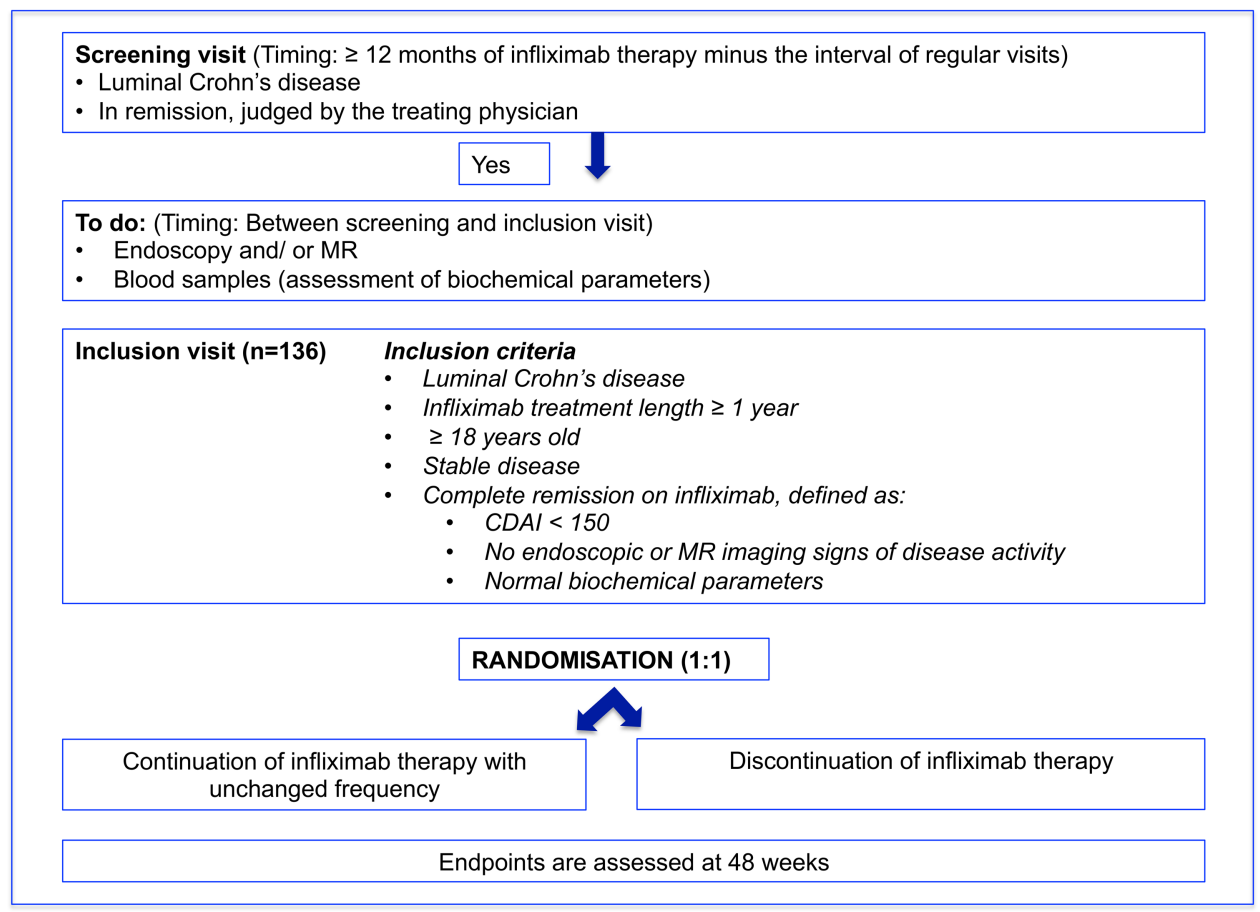

Figure 1 Study algorithm. 
Table 1 Course of study and data collection in the intervention and control groups

\begin{tabular}{|c|c|c|c|c|c|c|c|c|c|}
\hline Visit number & $\begin{array}{l}1 \\
\text { Screening }\end{array}$ & $\begin{array}{l}2 \\
\text { Inclusion* }\end{array}$ & 3 & 4 & 5 & 6 & 7 & 8 & 9 \\
\hline Week (from inclusion) & - & 0 & 4 & 8 & 16 & 24 & 32 & 40 & 48 \\
\hline Informed consent $†$ & $\square$ & & & & & & & & \\
\hline Endoscopy and/or MRI & $\square \ddagger$ & & & & & & & & $\square \ddagger$ \\
\hline Registration of demographics and medical history & & $\square$ & & & & & & & \\
\hline $\begin{array}{l}\text { Registration of adverse events and changes in } \\
\text { concomitant medication }\end{array}$ & $\square$ & $\square$ & $\square$ & $\square$ & $\square$ & $\square$ & $\square$ & $\square$ & $\square$ \\
\hline Focused physical examination & & $\square$ & $\square$ & $\square$ & $\square$ & $\square$ & $\square$ & $\square$ & $\square$ \\
\hline Pregnancy test & $\square$ & & & & & & & & \\
\hline $\begin{array}{l}\text { Biochemical parameters } \S \\
\text { - Blood sample } \\
\text { - Faecal sample }\end{array}$ & $\square$ & $\square$ & $\square$ & $\square$ & $\square$ & $\square$ & $\square$ & $\square$ & $\square$ \\
\hline Study medication & & $\square$ & & $\square$ & $\square$ & $\square$ & $\square$ & $\square$ & \\
\hline Blood sample before infusionף & $\square^{* *}$ & $\square$ & $\square$ & $\square$ & $\square$ & $\square$ & $\square$ & $\square$ & $\square$ \\
\hline $\begin{array}{l}\text { Blood sample after infusion (other arm) and } 1 \mathrm{~h} \\
\text { after the end of infusion? }\end{array}$ & $\square^{\star *}$ & $\square$ & & $\square$ & $\square$ & $\square$ & $\square$ & $\square$ & \\
\hline
\end{tabular}

Index (WPAI) ${ }^{29}$ life quality score (IBDQ short), ${ }^{30} 31$ endoscopy (Simple Endoscopic Score for Crohn's disease (SES-CD)) and/or MRI, and biochemical parameters at the time of inclusion. At the inclusion visit, patients are randomised (block randomisation and an allocation ratio of 1:1) to either continue IFX therapy at an unchanged dosage, or alternatively to receive matching placebo. Randomisation is stratified according to concomitant immunosuppressive therapy and history of fistulising disease. Randomisation is done centrally. The allocation sequence is in opaque, sealed envelopes. A non-blinded nurse, who is not involved in the treatment of the patient, receives the allocation result and subsequently prepares and labels IFX or placebo medication accordingly. Patients, treating nurses and treating physicians are blinded as to the type of medication (IFX or placebo) which the patient receives.

Following the screening and inclusion visit, patients are seen after 4 weeks; thereafter, the next consultations will be undertaken as part of the regular visits related to control, every 8 weeks. Disease activity will be assessed at every study-related visit by CDAI, WPAI, IBDQ and by biochemical parameters.

The study is terminated after 48 weeks (visit 9). At the time of visit 9, all patients will be graded for disease activity in accordance with CDAI, WPAI, IBDQ short, biochemical parameters and by endoscopic examination (SES-CD)) and/or MRI. The treating physician and the patient will, after the termination of the study, be informed about whether the patient has continued IFX or received a placebo.

Patients who relapse during the course of the study will be withdrawn and treated at the discretion of the treating physician. It is the intention that patients who were randomised to discontinue IFX and subsequently relapse will be offered inclusion in an open label study of the effect of retreatment with IFX. This is, however, a separate study, not a part of the present study.

\section{End points}

The primary end point of this study is the proportion of patients who maintain remission at the end of the trial after 48 weeks. Remission is defined as $\mathrm{CDAI}<150$.

Secondary end points assessed at the end of the trial after 48 weeks include:

- Proportion of patients who maintain complete remission (def. CDAI score $<150$, no signs of inflammation on biochemical parameters and mucosal healing).

- Median time to relapse after discontinuation of IFX.

- Proportion of patients experiencing relapse. Relapse, defined as a CDAI score $>150$ and a greater than 70 point increase from inclusion over two consecutive weeks. ${ }^{9} 31$

- Change from baseline in disease activity (ie, CDAI score, biochemical parameter including f-calprotectin, SES-CD).

- Economical expenses for treatment of Crohn's disease in the two groups.

\section{Sample size}

Calculations are based on the assumption that remission rates are higher in patients maintaining IFX treatment compared to patients receiving a placebo. Relapse rates in patients during ongoing IFX therapy was estimated to be $13 \%$ per year in a recent review by Gisbert and Panés. ${ }^{18}$ As we will include a selected group of patients who have already received treatment for a year with good response, we expect the remission rate in patients 
who continue IFX maintenance therapy to be $90 \%$. In the STORI study, the subgroup with the best prognostic markers had a similar proportion of patients maintaining remission. However, it is unknown if this can be extrapolated to other populations, especially because no control group was included in the STORI study. ${ }^{12}$ Thus, remission rates on discontinuation may be somewhat lower. A difference between patients continuing and patients stopping IFX of 20 percentage points is considered clinically relevant. Based on this minimally clinically relevant difference of $20 \%$ and an $\alpha$ set at 0.05 (two sided) and a $\beta$ set at 0.2, (IBM SPSS Sample Power V.3), a total of 62 patients in each group are needed to demonstrate a clinically relevant difference of continuing versus discontinuing IFX therapy. To correct for dropouts, it is planned to include 136 patients in the study.

\section{Data analysis plan}

The inclusion period extends from 1 November 2012 until 31 October 2015. The study completion is scheduled for November 2016. Thereafter, we will analyse the data and present results for scientific publications. In order to obtain adequate participant enrolment, the study employs multiple centres. The study is currently ongoing at five Danish sites and remains open for participation by new centres. The list of study sites can be viewed at clinical.trial.org.

Statistical analysis plan: Descriptive statistics will be calculated as percentages for discrete variables, and median with IQR or mean with SEM for continuous variables, as appropriate. Fisher's exact or $\chi^{2}$ test, as appropriate, will be used for univariate analysis of discrete variables. Unpaired $\mathrm{t}$ test/paired $\mathrm{t}$ test or Mann-Whitney $\mathrm{U}$ test/ Wilcoxon signed-rank test will be used for univariate analysis of continuous variables, as appropriate. Time until relapse will be estimated using survival statistics, that is, the Kaplan-Meier method and log-rank test. Association of demographical, clinical and biochemical variables with relapse will be estimated using univariate and multivariabel Cox proportional hazard regression analysis. $p$ Values are two sided, and $\mathrm{p}<0.05$ is considered significant.

\section{Explorative analyses}

In order to investigate whether pharmacoimmunological data can rationalise therapeutic management with respect to continuation or discontinuation of IFX therapy, patients will, on the day of infusion, have three blood samples drawn: one just before infusion (trough), one right after the infusion (obtained from the other arm) (peak) and one an hour after infusion $\left(\mathrm{C}_{1}\right)$. Based on disease activity after 1 year, patients are categorised as either in complete remission or not in complete remission. Results of IFX (and anti-IFX Ab) trough, peak and $\mathrm{C}_{1}$ concentrations in the two groups (remitters vs nonremitters) will be compared. Samples will be measured by common solid and fluid phase assays for this purpose, for example, Reporter Gene Assay (RGA). ${ }^{162032} 33$

\section{ETHICS AND DISSEMINATION}

\section{Ethical and safety considerations}

It is essential to determine strategies for discontinuation of IFX therapy in patients with Crohn's disease. Cessation of IFX therapy must not provide a very high risk of the disease to flare. On the other hand, the possible risk for development of lymphoma, including hepatosplenic T-cell lymphoma, which is often fatal, and other malignancies cannot be excluded. In particular, young patients and patients who have received prolonged treatment are at risk. ${ }^{34} 35$ In addition, though seldom, severe side effects to IFX present a danger for patient safety.

Patients participating in this study may potentially benefit from the discontinuation if they are able to tolerate cessation. The cessation can continue after the study period, as long as they experience low disease activity. In case of relapse, after discontinuation of IFX, data from our study ${ }^{14}$ and from the STORI study ${ }^{12}$ suggest that the patient will respond very well to retreatment. Patients who have been randomly selected to continue IFX therapy are subjected to the same side effects as they would have faced before entering the study. Participation in the trial should provide new data and information to benefit future patients.

No study-related activities will be performed until informed consent has been obtained. Investigators are responsible for obtaining written consent from each patient. During the trial, the patients will be monitored closely and every reasonable precaution will be taken to ensure patients' safety. At each visit, adverse events (AEs) will be elicited, and in case of any AEs a description will be recorded including intensity and causal relationship to the study drug. The principal investigator must inform the Danish Medicines Agency immediately if suspected unexpected serious adverse reactions (SUSARs) occur during the trial. Investigators must immediately report all serious incidents to the principal investigator. In case of a serious AE or SUSAR, the blinding for the involved patient is ceased. Patients will be informed if there are major changes to the trial. Investigators will be informed if there are important protocol modifications. The trial will be prematurely terminated if scientific findings from other research groups eliminate the trial. The trial will also be disrupted if there are serious, not yet known, side effects to the drugs used. IFX is a registered drug and is administered following the current instructions for administering the drug. Facilities for resuscitation and intensive therapy will be immediately at hand and patients should be under observation during the infusion.

The project will provide new knowledge regarding how to optimally handle patients with Crohn's disease in sustained remission on a TNFi, and will help develop new therapeutic strategies for this patient group. The study will provide information about the value of measuring concentrations of TNFi and antibodies against TNFi in an everyday clinical decision-making set-up. In 
addition, anti-TNF treatment plays an important role in many inflammatory diseases (eg, rheumatoid arthritis, spondylarthritis and psoriasis). ${ }^{36} 37$ As is the case in patients with Crohn's disease, discontinuation of TNFi in patients with other inflammatory diseases-after achievement of low disease activity-is important for reasons of safety and economy. ${ }^{38}$ Thus, the study results might inspire and expand similar research studies in other inflammatory diseases impacting patient safety of TNFi therapies.

\section{Publication}

It is the intention that any results, positive, negative or inconclusive, will be published in a relevant English language scientific journal/conference. SSB will be the first author. The last authors will be MA, KB, JB, OØT and $\mathrm{CS}$, the order of which will depend on the specific contributions in each manuscript. The site investigators will be the coauthors.

\section{Data deposition and curation}

We will store biological material in the form of blood samples from the patients. The samples are stored for 10 years after the completion of the study, after which all samples and leftover material will be destroyed. Patient-identifiable information (eg, name) will be treated as strictly confidential and will not be made publicly available. All information about the patients, including nonclinical data, protocols, case report forms and verbal and written information, are protected under the act concerning the processing of personal data and the Danish health law. Investigators will have access to the final trial data set. The study will comply with the ICH-GCP (Good Clinical Practice) rules and be performed in accordance with the protocol and relevant regulatory requirements.

Contributors All authors have been involved in the conception and design of the study. All authors contributed to the refinement of the study protocol. SSB, MAA and CS performed the drafting of the protocol manuscript. JB, $0 \varnothing \mathrm{T}$ and $\mathrm{KB}$ have been involved in the revision of the manuscript. All authors approve the final manuscript and agree to be accountable for all aspects of the work in ensuring that questions related to the accuracy or integrity of any part of the work will be appropriately investigated and resolved. They also participated in the implementation of the study.

Funding This study is supported by Nordforsk, Herlev Hospital Research Council, Danish Colitis-Crohn Society, and A.P. Møller and Chastine Mc-Kinney Møller's Foundation for General Purposes.

Competing interests CS has served as a speaker for MSD and Abbvie and as a consultant for MSD and Takeda Pharmaceutical Company. JB has served as an advisory board member for Abbvie. ОØT has served as a speaker and consultant for UCB and Zealand Pharma, speaker for MSA, and primary investigator for Amgen, Biogen, Novo-Nordisk and Pfizer. KB has served as a speaker for Pfizer, Roche, Novo-Nordisk, Bristol-Meyers Squibb and Biomonitor and owns stocks in Novo-Nordisk and Biomonitor

Patient consent Obtained.

Ethics approval The Danish Medicines Agency (EudraCT-number: 2012-002702-51). The Regional Ethics Committee of Region Hovedstaden Denmark (Approval-number: H-4-2012-099). The Danish Data Protection Agency (ID-number: 2007-58-0015/HEH.750.89-27).
Provenance and peer review Not commissioned; externally peer reviewed.

Data sharing statement The study is registered at Clinicaltrials.gov (http:// clinicaltrials.gov/show/NCT01817426).

Open Access This is an Open Access article distributed in accordance with the Creative Commons Attribution Non Commercial (CC BY-NC 4.0) license, which permits others to distribute, remix, adapt, build upon this work noncommercially, and license their derivative works on different terms, provided the original work is properly cited and the use is non-commercial. See: http:// creativecommons.org/licenses/by-nc/4.0/

\section{REFERENCES}

1. Talley NJ, Abreu MT, Achkar JP, et al. An evidence-based systematic review on medical therapies for inflammatory bowel disease. Am J Gastroenterol 2011;106(Suppl 1):S2-25; quiz S26.

2. Clark M, Colombel JF, Feagan BC, et al. American gastroenterological association consensus development conference on the use of biologics in the treatment of inflammatory bowel disease, June 21-23, 2006. Gastroenterology 2007;133:312-39.

3. Dignass A, Van Assche G, Lindsay JO, et al. The second European evidence-based Consensus on the diagnosis and management of Crohn's disease: current management. J Crohns Colitis 2010:4:28-62

4. Travis SP, Stange EF, Lémann M, et al. European evidence-based consensus on the management of ulcerative colitis: current management. J Crohns Colitis 2008;2:24-62.

5. D'Haens GR, Panaccione R, Higgins PDR, et al. The London Position Statement of the World Congress of Gastroenterology on Biological Therapy for IBD with the European Crohn's and Colitis Organization: when to start, when to stop, which drug to choose, and how to predict response? Am J Gastroenterol 2011;106:199-212; quiz 213.

6. Louis E, Belaiche J, Reenaers C. Anti-TNF and Crohn's disease: when should we stop? Curr Drug Targets 2010;11:148-51.

7. Danese S, Colombel JF, Reinisch W, et al. Review article: IFX for Crohn's disease treatment-shifting therapeutic strategies after 10 years of clinical experience. Aliment Pharmacol Ther 2011;33:857-69.

8. Clarke K, Regueiro M. Stopping immunomodulators and biologics in inflammatory bowel disease patients in remission. Inflamm Bowel Dis 2012;18:174-9.

9. Steenholdt C, Svenson M, Bendtzen K, et al. Severe infusion reactions to IFX: aetiology, immunogenicity and risk factors in patients with inflammatory bowel disease. Aliment Pharmacol Ther 2011;34:51-8.

10. Steenholdt $\mathrm{C}$, Svenson M, Bendtzen $\mathrm{K}$, et al. Incidence of acute severe infusion reactions to IFX depends on definition used rather than assay: authors' reply. Aliment Pharmacol Ther 2011;34:404-5.

11. Mowat C, Cole A, Windsor A, et al. Guidelines for the management of inflammatory bowel disease in adults. Gut 2011;60:571-607.

12. Louis E, Mary JY, Vernier-Massouille G, et al. Maintenance of remission among patients with Crohn's disease on antimetabolite therapy after IFX therapy is stopped. Gastroenterology 2012;142:63-70.e5; quiz e31.

13. Molnár T, Lakatos PL, Farkas K, et al. Predictors of relapse in patients with Crohn's disease in remission after 1 year of biological therapy. Aliment Pharmacol Ther 2013;37:225-33.

14. Steenholdt C, Molazahi A, Ainsworth MA, et al. Outcome after discontinuation of IFX in patients with inflammatory bowel disease in clinical remission: an observational Danish single center study. Scand J Gastroenterol 2012;47:518-27.

15. Solberg IC, Vatn MH, Høie O, et al. Clinical course in Crohn's disease: results of a Norwegian population-based ten-year follow-up study. Clin Gastroenterol Hepatol 2007;5:1430-8.

16. Baert F, Noman M, Vermeire $S$, et al. Influence of immunogenicity on the long-term efficacy of IFX in Crohn's disease. N Engl J Med 2003;348:601-8.

17. Sostegni R, Daperno M, Scaglione N, et al. Crohn's disease: monitoring disease activity. Aliment Pharmacol Ther 2003;17:11-17.

18. Gisbert JP, Panés J. Loss of response and requirement of IFX dose intensification in Crohn's disease: a review. Am J Gastroenterol 2009;104:760-7.

19. Steenholdt C, Bendtzen K, Brynskov J, et al. Cut-off levels and diagnostic accuracy of IFX trough levels and anti-IFX antibodies in Crohn's disease. Scand J Gastroenterol 2011;46:310-18.

20. Bendtzen K, Ainsworth M, Steenholdt C, et al. Individual medicine in inflammatory bowel disease: monitoring bioavailability, pharmacokinetics and immunogenicity of anti-tumour necrosis factor-alpha antibodies. Scand J Gastroenterol 2009;44:774-81. 
21. Influence of Immunogenicity on the Long-Term Efficacy of IFX in Crohn's Disease. Influence of Immunogenicity on the Long-Term Efficacy of IFX in Crohn's Disease.

22. Steenholdt C, Al-Khalaf M, Brynskov J, et al. Clinical implications of variations in anti-IFX antibody levels in patients with inflammatory bowel disease. Inflamm Bowel Dis 2012;18:2209-17.

23. Munkholm $\mathrm{P}$, Langholz $\mathrm{E}$, Nielsen $\mathrm{OH}$, et al. Incidence and prevalence of Crohn's disease in the county of Copenhagen 1962-87: a sixfold increase in incidence. Scand J Gastroentero 1992;27:609-14.

24. Sipponen $\mathrm{T}$, Nuutinen $\mathrm{H}$, Turunen $\mathrm{U}$, et al. Endoscopic evaluation of Crohn's disease activity: Comparison of the CDEIS and the SES-CD [Internet]. Inflamm Bowel Dis 2010;16:2131-6.

25. Daperno M, D'Haens G, Van Assche G, et al. Development and validation of a new, simplified endoscopic activity score for Crohn's disease: the SES-CD. Gastrointest Endosc 2004;60:505-12.

26. Grand DJ, Kampalath V, Harris A, et al. MR enterography correlates highly with colonoscopy and histology for both distal ileal and colonic Crohn's disease in 310 patients. Eur J Radiol 2012;81: e763-9.

27. Tolan DJ, Greenhalgh R, Zealley IA, et al. MR enterographic manifestations of small bowel Crohn disease. Radiographics 2010;30:367-84.

28. Best WR, Becktel JM, Singleton JW, et al. Development of a Crohn's disease activity index. National Cooperative Crohn's Disease Study. Gastroenterology 1976;70:439-44.

29. Reilly MC, Gerlier L, Brabant Y, et al. Validity, reliability, and responsiveness of the work productivity and activity impairment questionnaire in Crohn's disease. Clin Ther 2008;30:393-404.

30. Irvine EJ, Zhou Q, Thompson AK. The Short Inflammatory Bowel Disease Questionnaire: a quality of life instrument for community physicians managing inflammatory bowel disease. CCRPT Investigators. Canadian Crohn's Relapse Prevention Trial. Am J Gastroenterol 1996;91:1571-8.

31. Guyatt G, Mitchell A, Irvine EJ, et al. A new measure of health status for clinical trials in inflammatory bowel disease. Gastroenterology 1989;96:804-10.

32. Steenholdt C. Comparison of techniques for monitoring IFX bioavailability and immunogenicity in Crohn's disease. Ther Drug Monit 2013;35:530-8.

33. Lallemand C, Kavrochorianou N, Steenholdt C, et al. Reporter gene assay for the quantification of the activity and neutralizing antibody response to TNF $\alpha$ antagonists. J Immunol Methods 2011;373:229-39.

34. Peyrin-Biroulet L, Deltenre P, de Suray N, et al. Efficacy and safety of tumor necrosis factor antagonists in Crohn's disease: meta-analysis of placebo-controlled trials." Clin Gastroenterol Hepatol 2008;6:644-53.

35. Mackey AC, Green L, Liang LC, et al. Hepatosplenic T cell lymphoma associated with IFX Use in young patients treated for inflammatory bowel disease. J Pediatr Gastroenterol Nutr 2007;44:265-7.

36. Singh JA, Christensen R, Wells GA, et al. A network meta-analysis of randomized controlled trials of biologics for rheumatoid arthritis: a Cochrane overview. CMAJ 2009;181:787-96.

37. van der Heijde D, Sieper J, Maksymowych WP, et al. 2010 Update of the international ASAS recommendations for the use of anti-TNF agents in patients with axial spondyloarthritis. Ann Rheum Dis 2011;70:905-8.

38. Harigai M, Takeuchi T, Tanaka $\mathrm{Y}$, et al. Discontinuation of adalimumab treatment in rheumatoid arthritis patients after achieving low disease activity. Mod Rheumatol 2012;22:814-22. 